CARDIOVASCULAR MEDICINE

\title{
Systematic review of multidisciplinary interventions in heart failure
}

\author{
R Holland, J Battersby, I Harvey, E Lenaghan, J Smith, L Hay
}

Heart 2005;91:899-906. doi: 10.1136/hrt.2004.048389

See end of article for authors' affiliations

....................

Correspondence to: Dr Richard Holland, School of Medicine, Health Policy and Practice, University of East Anglia, Norwich, Norfolk NR4 TTJ, UK; r.holland@uea. ac.uk

Accepted

8 November 2004

\begin{abstract}
Objective: To determine the impact of multidisciplinary interventions on hospital admission and mortality in heart failure.

Design: Systematic review. Thirteen databases were searched and reference lists from included trials and related reviews were checked. Trial authors were contacted if further information was required.

Setting: Randomised controlled trials conducted in both hospital and community settings.

Patients: Trials were included if all, or a defined subgroup of patients, had a diagnosis of heart failure. Interventions: Multidisciplinary interventions were defined as those in which heart failure management was the responsibility of a multidisciplinary team including medical input plus one or more of the following: specialist nurse, pharmacist, dietician, or social worker. Interventions were separated into four mutually exclusive groups: provision of home visits; home physiological monitoring or televideo link; telephone follow up but no home visits; and hospital or clinic interventions alone. Pharmaceutical and exercise based interventions were excluded.

Main outcome measures: All cause hospital admission, all cause mortality, and heart failure hospital admission.

Results: 74 trials were identified, of which 30 contained relevant data for inclusion in meta-analyses. Multidisciplinary interventions reduced all cause admission (relative risk (RR) $0.87,95 \%$ confidence interval (Cl) 0.79 to $0.95, p=0.002$ ), although significant heterogeneity was found ( $p=0.002$ ). All cause mortality was also reduced (RR $0.79,95 \% \mathrm{Cl} 0.69$ to $0.92, p=0.002$ ) as was heart failure admission (RR $0.70,95 \% \mathrm{Cl} 0.61$ to $0.81, \mathrm{p}<0.001$ ). These results varied little with sensitivity analyses. Conclusion: Multidisciplinary interventions for heart failure reduce both hospital admission and all cause mortality. The most effective interventions were delivered at least partly in the home.
\end{abstract}

$\mathrm{H}$ eart failure is a common and serious public health problem. Recent estimates suggest a prevalence of congestive heart failure in Europe of $2.3 \% .{ }^{1}$ There has been a steady increase in hospital admissions for heart failure with almost 80000 recorded in the UK in 2000. The estimated total National Health Service (NHS) cost associated with heart failure in 2000 was $£ 905$ million-that is, $1.9 \%$ of NHS expenditure. ${ }^{2}$

Despite progress in heart failure treatments, such as increasing use of angiotensin converting enzyme (ACE) inhibitors and more recently $\beta$ blockade, many agree that the overall management of heart failure can be improved. ${ }^{34}$ Since 1999, several reviews of heart failure disease management programmes have been published, including two metaanalyses..$^{5-7}$ All reviews have concluded that specialised follow up of patients by a multidisciplinary team can reduce hospitalisation but have been unclear about the effect on survival. Since the most recent reviewers completed their search $^{6}$ a further 11 randomised trials have reported results. These have substantially increased the number of randomly assigned patients from 3304 to 8158 . It is therefore timely to review this larger body of evidence with its potential to provide more precise effect size estimates. Furthermore, we wished to investigate the relative merits of home, telemonitoring, telephone, and clinic or hospital based interventions. This question of the location of follow up has not been examined to date.

Home visits have the potential to allow educational interventions to be tailored to a patient's social circumstances. Telemonitoring gives people the freedom of their homes while permitting daily (or even continuous) evaluation of physiological state and often face to face contact through videophone technology. Telephone follow up allows hospital advice to be re-enforced and promotes continuity of care $^{8}$ but, although less time consuming, it is also less personal than a home visit or telemonitoring.

The objective of this systematic review of all available randomised controlled trials was to determine the impact of multidisciplinary interventions on hospital admission and mortality in patients with heart failure. Multidisciplinary interventions were defined as those in which management was the responsibility of a multidisciplinary team that included medical input plus one or more of the following: a specialist nurse, a pharmacist, a health educator, a dietician, or a social worker. Trials of drugs, pacemakers, mechanical appliances, and exercise-alone interventions were excluded.

\section{METHODS}

\section{Data sources}

The search strategy identified research on patients with congestive heart failure that tested multidisciplinary interventions and reported outcomes of admission, mortality, or quality of life. Multidisciplinary interventions were identified by a broad range of search terms and medical subject headings: home care services, patient or health education, community health nursing, patient care planning,

\footnotetext{
Abbreviations: ACE, angiotensin converting enzyme; AMED, Allied and Complementary Medicine; $\mathrm{Cl}$, confidence interval; $\mathrm{CINAHL}$, Cumulative index to Nursing and Allied Health Literature; $\mathrm{COACH}$, coordinating study evaluating outcomes of advising and counselling in heart failure; DARE, Database of Abstracts of Reviews of Effects; NHS, National Health Service; TEN-HMS, Trans-European Network initiativehomecare management system study
} 
pharmaceutical services, and telemonitoring. This search was developed with reference to the indexing of previously identified studies. The following electronic databases were searched from their inception to I June 2004: Medline, Embase, Cumulative index to Nursing and Allied Health Literature (CINAHL), Allied and Complementary Medicine (AMED), Cochrane Controlled Trials Register, the Cochrane Effective Practice and Organization of Care Study Registry, Biomed, the TRIP Database, Meta-Register of Current Controlled Trials, Research Findings Electronic Register, NHS Research Register, the Cochrane Database of Systematic Reviews, and the Database of Abstracts of Reviews of Effects (DARE). Reference lists of all included articles and relevant review articles were searched. There were no language restrictions.

\section{Study selection}

One of three investigators (JB, RH, and EL) reviewed all titles retrieved by the literature search. Titles needed to appear potentially relevant to the study area. Two investigators (JB and RH) independently assessed abstracts against three criteria to determine, firstly, that the study was a randomised controlled trial; secondly, that a defined group of patients were identified as having heart failure; and thirdly, that the intervention was non-pharmaceutical and non-exercise based. Interventions needed to be applied to patients and not focus solely on changing physician management. Trials incorporating exercise advice among a range of other heart failure issues were included. Multidisciplinary interventions were nurse led programmes, medication review, medication adherence interventions, patient education, or enhanced monitoring. Studies could be carried out in any setting (hospital, clinic, home, etc). Full papers were retrieved if one or both investigators considered the abstract suitable. The two investigators then independently assessed retrieved studies for their suitability for inclusion in the meta-analysis. Differences were resolved by discussion with reference to a third reviewer (IH) if necessary.

\section{Data extraction}

Data were extracted (JB, RH, IH, EL) on to an electronic database based on a previously piloted data extraction form. Extracted data were type of participants, intervention details, outcomes, and trial quality characteristics.

\section{Classification of interventions}

Two researchers (JS and LH), who were blinded to all trial results, independently classified trial interventions and then agreed their decisions jointly. Studies were classified into one or more of the following definitions:

(1) One or more planned intervention visits in the patient's home by a health care professional to educate or improve patient self management (this excludes visits to take blood samples, set up physiological monitoring, or carry out interventions such as wound care)

(2) Videophone use or any form of home physiological monitoring

(3) One or more planned telephone calls to the patient at home

(4) Educational or symptom self management mailings to the patient's home address

(5) No planned intervention home visits; instead, intervention was provided during hospital admission or during hospital clinic attendance

(6) No planned intervention home visits; instead, intervention was provided in general practice or a community clinic.
For the meta-analyses, each trial was categorised according to the uppermost category into which it fitted. Thus, a trial classified as having an intervention delivered in hospital (5) with additional planned telephone follow up (3) was classified as category 3. Furthermore, as only one trial was classified in category 4, this was merged with category 3. Similarly, categories 5 and 6 were merged. This resulted in four final intervention subgroups: subgroup A entailed at least some home visiting; subgroup B, home physiological monitoring or televideo contact; subgroup $\mathrm{C}$, telephone or mailing to the patient's home without home visiting; and subgroup D interventions were delivered exclusively in a hospital, clinic, or general practice. These four subgroups were mutually exclusive.

Trial interventions were also classified as high or low intensity. High intensity interventions consisted of planned contact at least monthly with multifaceted interventions; low intensity interventions entailed less frequent contact or narrow interventions (for example, medication review alone).

\section{Outcomes}

The primary outcome was the proportion of patients with one or more hospital admission (all cause). Secondary outcomes were all cause mortality, heart failure hospital admission, and mean inpatient days. Data were extracted from studies for their predefined final follow up. Data on quality of life were also extracted but are not presented here owing to the diversity of scales used, which prevented data pooling.

\section{Quality assessment}

Many quality scales have been created to judge trials. These scales often give importance to subject blinding, which was impossible within the trials considered by this review. Juni et $a l^{9}$ recommend estimating trial quality by assessing three key components: concealment of allocation, use of intention to treat analysis, and blinding of outcome assessment. Blinding of outcome assessment was rarely reported for our outcomes (hospital admission or death). We therefore assessed whether studies confirmed outcome data by using at least two sources (for example, hospital data and self report). In addition, trial quality was assessed against criteria recommended by the York Centre for Reviews and Dissemination ${ }^{10}$ : firstly, explicit statement of inclusion or exclusion criteria; secondly, baseline comparability between groups; and thirdly, a clearly defined primary outcome. The review team also considered the following criteria important in reference to this particular study area: clear diagnostic criteria for heart failure and length of follow up (where six months and over was considered adequate). This gave a total of eight quality criteria against which studies were assessed.

\section{Data synthesis}

All trials that reported appropriate data were included in the meta-analyses if interventions were compared with usual care. Authors of trials were contacted to confirm the data extraction and additional data were sought where articles did not report one or more outcomes. For data on proportion of patients admitted (all cause and heart failure) and mortality, the effect of the intervention was reported as a relative risk. For mean inpatient days a weighted mean difference was calculated. Meta-analyses were carried out with the random effects methods in RevMan version 4.2 (Cochrane Collaboration, Oxford, UK). Funnel plots were constructed to assess possible publication bias.

\section{Sensitivity analyses}

The robustness of the findings in relation to all cause admission and mortality was investigated in the following sensitivity analyses. Firstly, we investigated reasons for any 


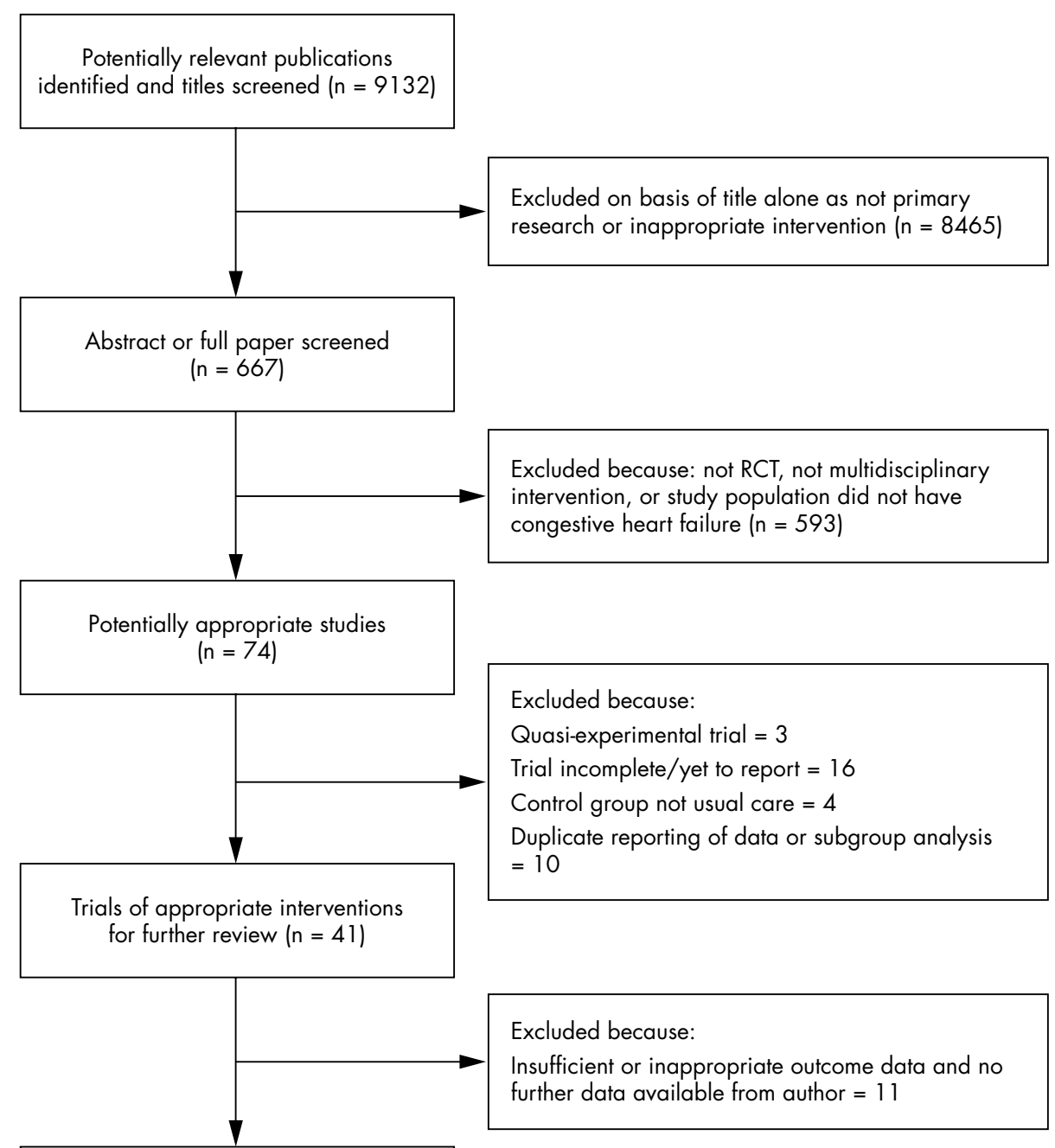

Figure 1 Flowchart describing study selection and excluded studies. RCT, randomised controlled trial.

\footnotetext{
Trials with outcome data useful in meta-analysis (total $=30$ )

- 21 all-cause re-admission data

- 27 all-cause mortality data

- 16 heart failure re-admission data

- 10 mean inpatient days
}

heterogeneity found and repeated analyses with fixed effects methods. Next we explored the effect of excluding poorer quality studies. This was investigated in two ways: by removing those lacking components recommended by Juni et $\mathrm{al}^{9}$ and by removing those achieving $50 \%$ or less of the eight criteria above. Trials where randomisation was not of individual patients (that is, cluster trials) were also excluded from analysis. These report overprecise results if adjustment is not made for the clustering of data. In the context of a meta-analysis such data can potentially bias results. Analyses were also repeated excluding trials yet to formally publish results in a peer reviewed article. Lastly, analyses were repeated excluding trials that recruited broadly, in which patients with heart failure were simply a subgroup.

\section{RESULTS}

\section{Search results}

We reviewed 9132 titles, which yielded 667 potentially relevant studies. Seventy four studies met the original inclusion criteria (fig 1). Of these, 21 contained sufficient published data to be included in the meta-analysis and a further nine were included after contact with authors yielded additional data. ${ }^{81-18}$ Forty four studies were excluded. Figure 1 lists reasons for exclusion.

\section{Study characteristics}

Of the 30 trials included in this review 13 were performed in Europe, 13 in the USA, three in Australia or New Zealand, and one in Argentina (table 1; to view table 1 visit the Heart website-http://www.heartjnl.com/supplemental). Twenty two trials $(73 \%)$ were published in the past five years (1999-2004). The earliest included trial was published in 1993. ${ }^{19}$ Patient inclusion criteria were reasonably similar across all studies. Four trials recruited patients from a hospital clinic or general practice ${ }^{12} 20-22$ and all others recruited hospitalised (or recently hospitalised) patients admitted with a diagnosis of heart failure. In almost all studies patients were excluded if they were due to be discharged to a long term care facility, had some form of cognitive impairment or psychiatric disorder, or had a 
terminal disease or severe co-morbidity. Despite the apparently similar inclusion criteria the mean age of patients varied between 56-86 years and the proportion of men from 27-99\%. Fifteen trials recorded baseline ejection fractions, with trial means varying from $22-43 \%$. Eight studies focused their intervention on a category of more severe heart failure (for example, New York Heart Association functional class $\left.>\mathrm{II}^{15}\right)$.

\section{Trial quality}

Only six studies clearly satisfied all three key quality components (concealed allocation, intention to treat, and data cross checked). ${ }^{1123-27}$ However, when trials were considered against all eight quality criteria the majority (22 of 30) met at least five. Two trials achieved only two criteria, ${ }^{1228}$ five achieved three criteria, ${ }^{131619222930}$ and one achieved four criteria. ${ }^{31}$ It should be noted that for one study only an abstract was available, which limited its quality assessment. $^{13}$

\section{Interventions}

Twelve trials had a home based component (subgroup A) (table 2; to view table 2 visit the Heart website-http:// www.heartjnl.com/supplemental). Home interventions included between one and eight visits over trial follow up (mean of 0.6 visits for each month of follow up). In two trials patients were visited only if they could not attend a clinic. ${ }^{18} 25$ All but two of these 12 interventions also included telephone contact. ${ }^{12}{ }^{32}$ Three trials included remote physiological monitoring (subgroup B). Twelve trials had telephone interventions or letters to patients ${ }^{28}$ (subgroup C). Patients received between one and 17 telephone calls over follow up (mean 1.4 for each month of follow up). Three trials delivered interventions exclusively in hospital or clinic settings (subgroup D). ${ }^{24} 2930$

\section{Effect on all cause admission}

Twenty one trials provided data on all cause admission (fig 2). No data were available from home monitoring trials on this outcome. Meta-analysis showed a significant reduction in all cause admission (relative risk 0.87, 95\% confidence interval (CI) 0.79 to $0.95, \mathrm{p}=0.002$ ). However, significant heterogeneity $(p=0.002)$ was found. Subgroup analysis showed that home visit interventions reduced all cause admission to hospital (relative risk $0.80,95 \%$ CI 0.71 to 0.89 , p $<0.0001$ ). Telephone-type interventions also reduced admission but this finding was of borderline significance (relative risk 0.86, 95\% CI 0.73 to $1.02, \mathrm{p}=0.09$ ). Hospital based interventions had no effect (relative risk $0.99,95 \%$ CI 0.90 to $1.10, \mathrm{p}=0.56$ ). Heterogeneity was found among telephone-type interventions $(\mathrm{p}<0.001)$ but not within the two other subgroups.

\section{Effect on all cause mortality}

Twenty seven trials provided data on all cause mortality (fig 3). Meta-analysis showed a significant decrease in all cause mortality (relative risk 0.79 , $95 \%$ CI 0.69 to 0.92 ,

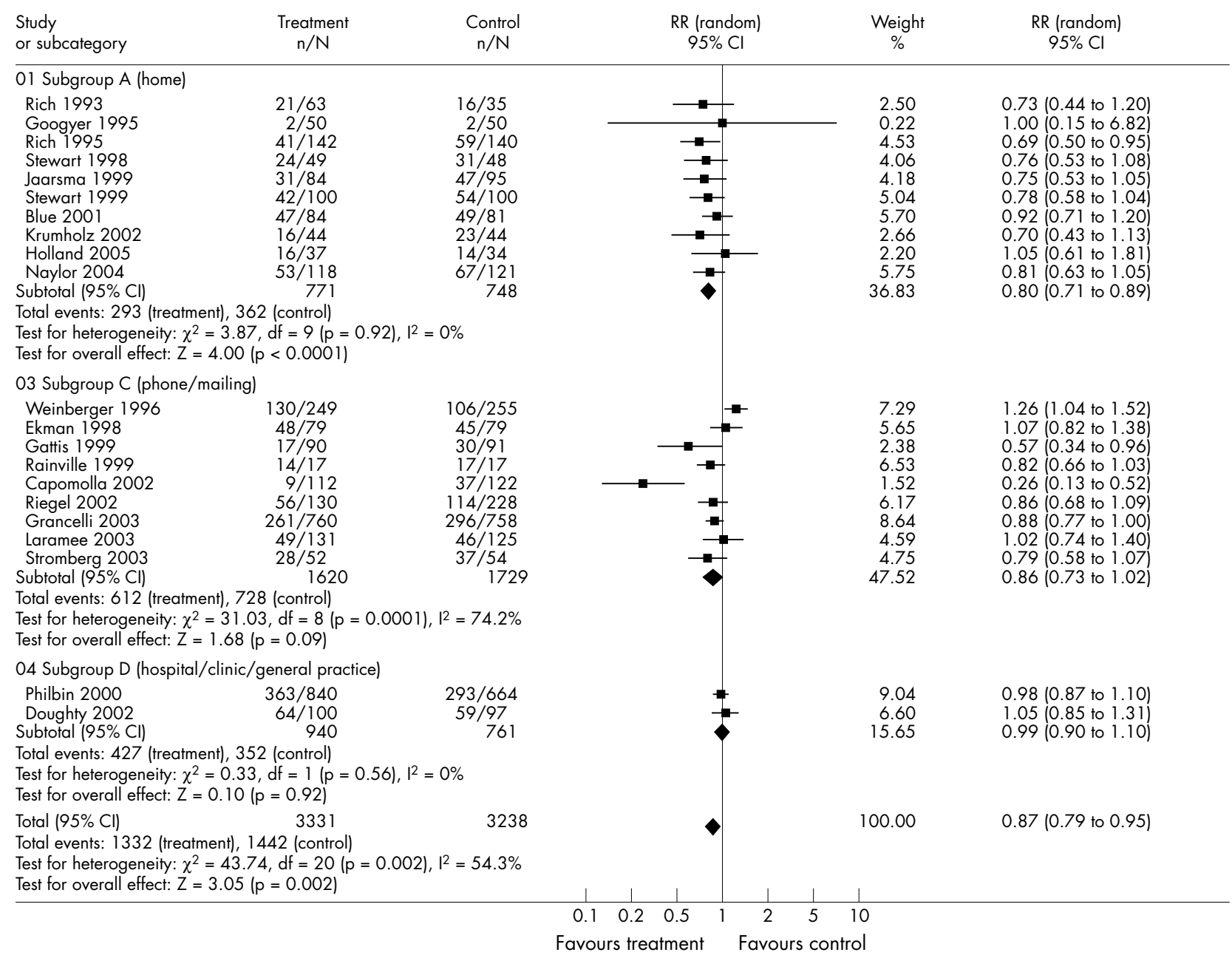

Figure 2 Forest plot for all cause admission results. Cl, confidence interval; RR, relative risk. Note: data on all cause admission were not available for the three trials in subgroup B (videophone/remote monitoring). 


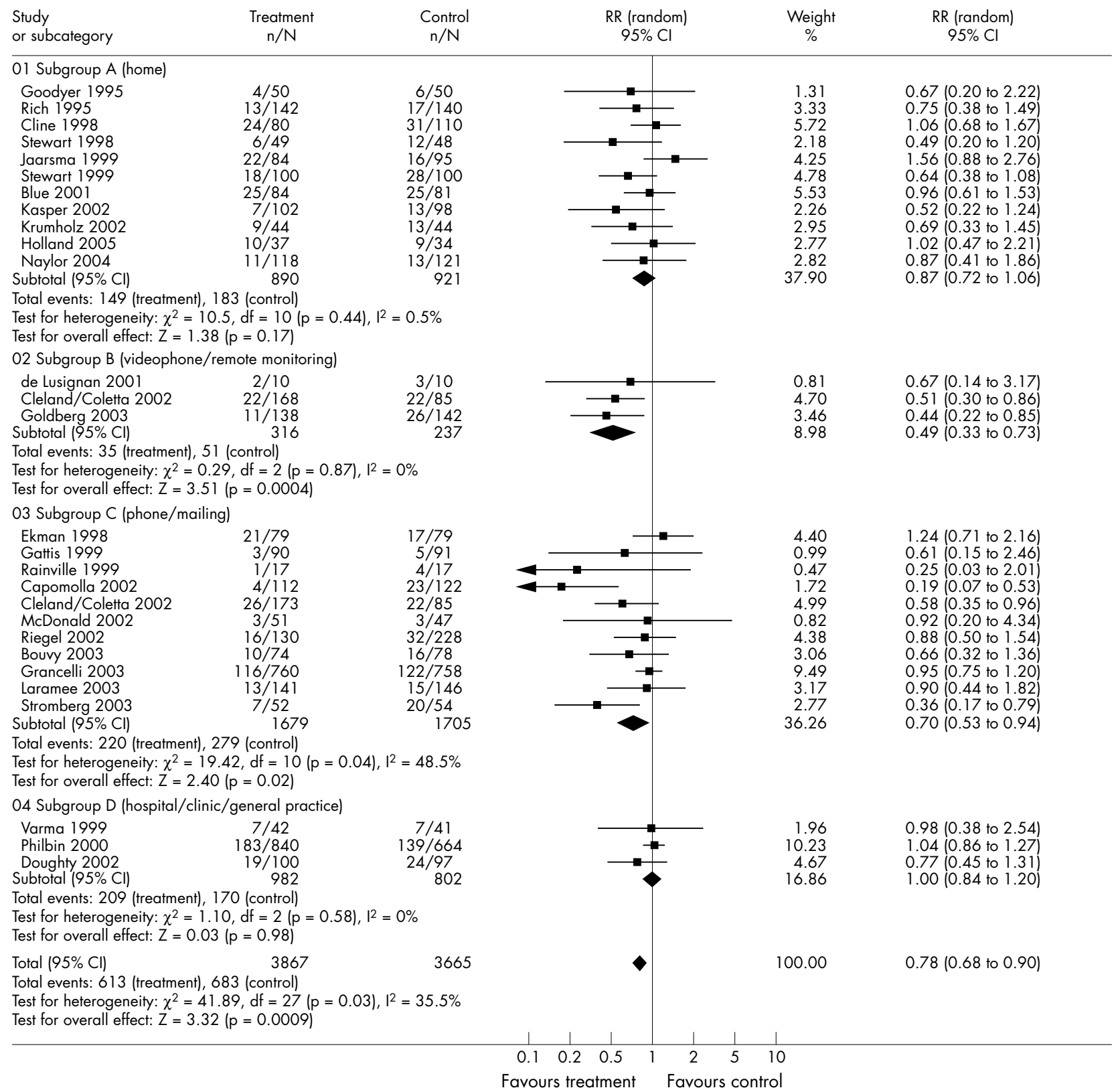

Figure 3 Forest plot of all cause mortality results. The control group from the study of Cleland/Coletta appears twice, as this was a three arm trial (telemonitoring, telephone support, and usual care). As a result, estimates for each subgroup are correctly calculated but the total estimate is slightly inaccurate. This should read 0.79 ( $95 \% \mathrm{Cl} 0.69$ to 0.92 ) and the overall test for heterogeneity should have a $\chi^{2}$ of $40.07, p=0.04$.

$\mathrm{p}=0.002)$. Subgroup analysis showed significant reductions in mortality for both telemonitoring (relative risk 0.49 , $95 \%$ CI 0.33 to $0.73, \mathrm{p}<0.001)$ and telephone follow up (relative risk $0.70,95 \%$ CI 0.53 to $0.94, p=0.02$ ). Reductions in mortality were non-significant in the home (relative risk $0.87,95 \%$ CI 0.72 to $1.06, \mathrm{p}=0.44$ ) and clinic subgroups (relative risk 1.00, 95\% CI 0.84 to $1.20, \mathrm{p}=0.98$ ). All subgroup confidence intervals overlapped, with the exception of subgroups B and D. Heterogeneity was detected for the overall result $(p=0.04)$ and for the telephone subgroup $(\mathrm{p}=0.04)$.

\section{Effect on heart failure admission}

Sixteen trials provided data on heart failure admission. Metaanalysis showed a significant reduction in heart failure admission with a relative risk of 0.70 (95\% CI 0.61 to 0.81 , $p<0.0001$ ). The benefit was notable and similar for home and telephone-type interventions, with relative risks of 0.62 (95\% CI 0.51 to $0.74, \mathrm{p}<0.001$ ) and 0.70 (95\% CI 0.57 to $0.85, \mathrm{p}<0.001)$, respectively. No significant effect was apparent in the two studies of patients receiving a hospital or community based intervention (relative risk 0.94, 95\% CI 0.78 to $1.13, \mathrm{p}=0.51) .{ }^{24}{ }^{29}$ No heterogeneity was detected within the subgroups, but it was detected for the overall result $(\mathrm{p}=0.04)$.

\section{Effect on total admissions and mean inpatient days}

Multidisciplinary interventions appear to reduce not only the proportion of patients admitted but also the total number of admissions. Across 18 of the studies the intervention groups had a mean admission rate of 21.8 admissions versus 29.0/ 1000 patient weeks for control groups. These data should be interpreted with caution, as formal pooling was not possible due to lack of standard deviation data. The 10 trials that 
provided mean inpatient days and standard deviation data (seven home visits, three telephone interventions) were formally pooled. This showed a significant decrease in overall mean inpatient days of 1.9 days in favour of the intervention (95\% C.I. 0.71 to $3.1, p=0.002$ ), with no overall heterogeneity $(p=0.24)$.

\section{Sensitivity analyses}

Table 3 describes the various sensitivity analyses conducted on the primary outcome (all cause admissions). The overall effect was slightly reduced by considering a fixed effect estimate (relative risk $0.90 v 0.87$ ). Both results were highly significant $(p=0.002)$. However, in both analyses significant heterogeneity was found. This was principally caused by two outliers. One suggested very strong benefit ${ }^{31}$ and the other potential harm. ${ }^{27}$ The study showing a very strong benefit provided intensive heart failure support through a day hospital. ${ }^{31}$ Patients could be managed there as day patients with intravenous treatment if necessary, and these events did not then count as admissions. Such care was not available in any other study. The second outlier recruited a wide spectrum of patients (with heart failure and other diagnoses) and delivered an intervention focused particularly on medication before and immediately after discharge. ${ }^{27}$ Removing these two studies, which provided qualitatively different interventions from all others, reduced heterogeneity notably $(p=0.39)$ but only slightly reduced the overall effectiveness estimate (relative risk 0.89).

Table 3 also shows that sensitivity analyses around quality components had little impact on the effectiveness estimate. The largest impact came from limiting the meta-analysis to studies that clearly used concealed allocation. In this case the relative risk was reduced to 0.92 . In contrast to this, when quality was considered across eight trial components higher quality studies (judged as achieving five or more components) showed increased effectiveness (relative risk $0.85 \mathrm{v}$
0.94). A similar result was found when cluster trials were excluded, indicating that their inclusion could only have biased results towards no effect. In one of these trials the analysis had accounted for clustering but adjusted data required for the meta-analysis could not be extracted. ${ }^{24} \mathrm{~A}$ final quality indicator used was in terms of peer review publication of data. Data from eight studies were considered "unpublished". In these cases, data were either provided directly by the author, extracted from conference abstracts, or from web based material. Relative risk estimates were identical between meta-analysis of these data and published data $(0.87 v 0.86)$.

Among those providing admission data, 12 trials had high intensity interventions. These studies were from all our intervention categories (that is, home, telemonitoring, telephone, and hospital). These appeared only marginally more effective than low intensity interventions (relative risk $0.86 v$ 0.88), with overlapping confidence intervals. A clearer though non-significant difference was evident when mortality was considered (relative risk $0.76 v 0.84$ ).

Three studies recruited patients with a variety of diagnoses and applied more generic interventions. ${ }^{1127}{ }^{32}$ Together these studies did not show a benefit within their subgroup of patients with heart failure (relative risk 1.02). In contrast, studies solely recruiting patients with heart failure found reduced risk of admission, with little difference between those recruiting high risk patients and those that were less selective (relative risk $0.82 \vee 0.86$ ).

Identical sensitivity analyses were also performed for mortality results (table 3 ). These found broadly similar results to those described above for all cause admission.

\section{Funnel plots}

Funnel plots (available from the authors) suggested little evidence of publication bias.

Table 3 Sensitivity analyses

\begin{tabular}{|c|c|c|c|c|c|c|}
\hline \multirow[b]{2}{*}{ Analysis } & \multicolumn{3}{|c|}{ All cause admission } & \multicolumn{3}{|l|}{ Mortality } \\
\hline & $\begin{array}{l}\text { No of patients } \\
\text { (no of trials) }\end{array}$ & $\operatorname{RR}(95 \% \mathrm{Cl})$ & $p$ Value & $\begin{array}{l}\text { No of patients } \\
\text { (no of trials) }\end{array}$ & $\operatorname{RR}(95 \% \mathrm{Cl})$ & p Value \\
\hline \multicolumn{7}{|l|}{ Random versus fixed effects } \\
\hline Random effects & $6569(21)$ & $0.87(0.79$ to 0.95$)$ & 0.002 & $7447(27)$ & 0.79 (0.69 to 0.92$)$ & 0.002 \\
\hline Fixed effects & $6569(21)$ & 0.90 (0.85 to 0.95$)$ & $<0.001$ & 7447 (27) & 0.85 (0.77 to 0.94$)$ & 0.001 \\
\hline \multicolumn{7}{|l|}{ Removing outliers } \\
\hline Base case & $6569(21)$ & 0.87 (0.79 to 0.95$)$ & 0.002 & 7447 (27) & 0.79 (0.69 to 0.92$)$ & 0.002 \\
\hline Removing extreme trials & 5831 (19) & $0.89(0.83$ to 0.94$)$ & $<0.001$ & $7213(26)$ & $0.83(0.73$ to 0.95$)$ & 0.006 \\
\hline \multicolumn{7}{|l|}{ Quality component } \\
\hline \multirow{2}{*}{$\begin{array}{l}\text { 1. Concealed allocation } \\
\text { No clear concealment }\end{array}$} & $2238(10)$ & $0.92(0.80$ to 1.05$)$ & 0.22 & $3161(11)$ & 0.87 (0.74 to 1.01$)$ & 0.07 \\
\hline & 4331 (11) & $0.82(0.73$ to 0.95$)$ & 0.01 & $4286(16)$ & $0.74(0.58$ to 0.94$)$ & 0.01 \\
\hline \multirow{2}{*}{ 2. $\operatorname{IT}_{\text {Unc }}$} & $5363(14)$ & 0.88 (0.79 to 0.99$)$ & 0.03 & 5589 (17) & $0.76(0.63$ to 0.91$)$ & 0.003 \\
\hline & $1206(7)$ & $0.83(0.73$ to 0.94$)$ & 0.002 & $1858(10)$ & $0.86(0.67$ to 1.12$)$ & 0.26 \\
\hline \multirow{2}{*}{$\begin{array}{l}\text { 3. Data } \\
\text { No c }\end{array}$} & 3859 (13) & $0.86(0.75$ to 0.99$)$ & 0.03 & 2587 (13) & $0.86(0.72$ to 1.03$)$ & 0.10 \\
\hline & 2710 (8) & $0.89(0.80$ to 0.98$)$ & 0.02 & 4860 (14) & 0.72 (0.69 to 0.92$)$ & 0.007 \\
\hline \multicolumn{7}{|l|}{ Overall quality score } \\
\hline High score $(>5$ of 8$)$ & $4577(16)$ & $0.85(0.75$ to 0.95$)$ & 0.005 & $4793(20)$ & $0.82(0.71$ to 0.96$)$ & 0.01 \\
\hline & $1992(5)$ & $0.94(0.86$ to 1.04$)$ & 0.50 & $2654(7)$ & $0.69(0.45$ to 1.05$)$ & 0.08 \\
\hline \multicolumn{7}{|l|}{ Level of randomisation } \\
\hline Individual patient only & $4510(18)$ & $0.84(0.75$ to 0.94$)$ & 0.002 & $5388(24)$ & $0.76(0.64$ to 0.90$)$ & 0.002 \\
\hline Cluster trial & 2059 (3) & 0.97 (0.89 to 1.07$)$ & 0.56 & 2059 (3) & $0.99(0.83$ to 1.18$)$ & 0.92 \\
\hline \multicolumn{7}{|l|}{ Origin of data } \\
\hline Published article & 4315 (13) & 0.86 (0.75 to 0.98$)$ & 0.02 & $4740(21)$ & 0.83 (0.81 to 0.97$)$ & 0.02 \\
\hline Data from author/abstract & 2254 (8) & $0.87(0.79$ to 0.95$)$ & 0.003 & $2707(6)$ & 0.70 (0.48 to 1.02$)$ & 0.06 \\
\hline \multicolumn{7}{|l|}{ Intensity } \\
\hline High & $3593(11)$ & $0.85(0.75$ to 0.97$)$ & 0.02 & $4550(15)$ & 0.76 (0.63 to 0.92$)$ & 0.005 \\
\hline Low & $2976(10)$ & $0.88(0.76$ to 1.01$)$ & 0.07 & 2897 (12) & $0.84(0.66$ to 1.06$)$ & 0.15 \\
\hline \multicolumn{7}{|l|}{ Target of intervention } \\
\hline High risk CHF patient & $1354(7)$ & $0.82(0.69$ to 0.96$)$ & 0.02 & $1586(7)$ & $0.81(0.58$ to 1.15$)$ & 0.25 \\
\hline Any CHF patient & 4543 (11) & $0.86(0.78$ to 0.96$)$ & 0.007 & 5693 (18) & $0.79(0.67$ to 0.94$)$ & 0.008 \\
\hline Variety of diagnoses & $672(3)$ & $1.02(0.72$ to 1.44$)$ & 0.91 & $168(2)$ & $0.73(0.36$ to 1.51$)$ & 0.40 \\
\hline
\end{tabular}




\section{DISCUSSION}

This systematic review shows that delivering multidisciplinary interventions to patients with heart failure not only reduces hospital admission but also is an effective method for reducing mortality. Our results show that the risk of all cause admission is reduced by about $13 \%$, mortality by as much as $20 \%$, and heart failure admission by $30 \%$. Results for all cause admission and mortality were little changed by a wide variety of sensitivity analyses that excluded trials on the basis of quality criteria or clustering of data. This is the first metaanalysis of this form of intervention to show a significant reduction in mortality, and it is important to note that the reduction is similar to that achieved by ACE inhibitors in heart failure. ${ }^{40}$

This study also investigated whether effectiveness varied according to where the interventions were carried out. These subgroup analyses generated less clear cut results. Home based interventions successfully reduced all cause admission, heart failure admission, and mean days in hospital. However, they led to a non-significant decrease in deaths. The latter result may simply reflect that insufficient patients have been enrolled in home based trials as yet $(n=1909)$. In contrast, telephone interventions decreased heart failure admission and deaths but led to a non-significant decrease in all cause admission. Remote televideo or physiological monitoring provides a newer form of intervention. A few trials of these interventions have now been conducted. Although this review suggests that these interventions may have an important effect on mortality, data were not available to investigate their effect on admission. We found only three trials where interventions were solely delivered in a hospital, clinic, or general practice. ${ }^{24} 29$ None of these studies reported significant benefits.

Studies that we reviewed had reasonably broad inclusion criteria such that the findings are of relevance to most hospitalised patients with heart failure. However, trials tended to restrict participation to patients with no important co-morbidity, terminal disease, confusion, or residence in a long term care facility. Thus, the benefits may not extend to these groups. Equally, it should be noted that trial populations were reasonably heterogeneous, which is likely to have contributed to heterogeneity in the results.

This review has been enhanced by the provision of extra unpublished data from some authors and data from abstracts of trials presented at international conferences. Exclusion of these non-peer reviewed data within our sensitivity analysis did not change the effectiveness estimates but did broaden the confidence limits.

Eleven studies were excluded because they did not report outcome data that could be used by this review. However, eight of these reported other outcomes favouring their intervention groups, while three reported no clear differences. Funnel plots suggested little evidence of publication bias. Nonetheless, given the numbers of patients now randomly assigned $(>8000)$, it seems unlikely that publication of small negative studies would have an important impact on any of the effectiveness estimates.

A diverse range of interventions could have met this review's inclusion criteria. However, in reality the trials that we found tested relatively similar interventions. Almost all interventions shared two key elements: one to one patient education concerning heart failure, medication, and diet and exercise advice; and symptom monitoring and self management advice. Education tended to be given over a number of encounters. It should be noted that 11 trials incorporated interventions that appeared to be of high intensity. Effectiveness estimates from these appeared to be only marginally greater than those of lower intensity interventions and the confidence intervals overlapped. Equally, targeting the intervention at high risk patients with heart failure did not seem important. It is also interesting to note that trial results do not appear to have changed over time despite increased use of ACE inhibitors and introduction of low dose $\beta$ blockade (fig 2). This may suggest that the effectiveness of multidisciplinary interventions is mediated not solely through better compliance with modern drug treatments but also through better symptom self management and lifestyle changes.

No trial included in this review provided any form of placebo intervention. It is difficult to imagine how this would be delivered in practice. It is therefore possible that the effectiveness observed is partly due to increased social contact. A more important limitation of much of the research to date has been the use of a single highly motivated specialist team. Replicating this in other settings, particularly in rural areas where distances to specialist centres are large, is likely to be difficult. It seems important to test whether less specialised nurses or community pharmacists are as effective as these highly specialised teams.

A few studies were excluded from this review because they compared two interventions and did not include a usual care subgroup. These shed some light on the importance of intervention intensity. Benatar et $a l^{41}$ compared telemonitoring with frequent home visiting (nine visits over five weeks, then as needed) of 216 patients over 12 months' follow up. This trial reported heart failure admission data and found a non-significant reduction from telemonitoring (38 admissions) compared with home visiting (63 admissions). Jerant et $a l^{42}$ randomly assigned 37 patients to telemonitoring, telephone follow up, or usual care (where all groups received two detailed home visits) and followed them up over six months. This study's sample size limited its findings. In total there were nine admissions in the telemonitoring group, five in the telephone group, and 15 in the usual care group $(\mathrm{p}=0.45)$. Harrison et $a l^{43}$ randomly assigned 192 patients to receive usual home nursing care, which consisted of assessment, monitoring and health teaching, or a transitional care intervention. The transitional care intervention was a comprehensive discharge programme with evidence based counselling and education. Harrison et $a l^{43}$ found a nonsignificant decrease in the proportion of patients admitted after the transitional care intervention. Finally, Coletta et al (in the TEN-HMS (Trans-European Network initiativehomecare management system study)), ${ }^{13}$ whose interim results are included in this review, compared usual care with two interventions: a telephone intervention and a televideo intervention. This study has found an improvement in mortality in both intervention groups, but only a small non-significant difference between these groups (13\% for telemonitoring $v 15 \%$ for telephone). With the exception of the study of Jerant et al, ${ }^{42}$ these studies suggest a small incremental effect of intensive interventions, in line with the finding from our review comparing high intensity interventions versus low intensity interventions. However, this difference, as well as its relative cost effectiveness, needs to be more clearly established. Ongoing trials will add to this analysis over the next three years including the large $\mathrm{COACH}$ (coordinating study evaluating outcomes of advising and counselling in heart failure) trial, ${ }^{44}$ which is randomly assigning 1050 patients to either usual care, basic education and support, or intensive education and support, as well as final results from the TEN-HMS study. ${ }^{13}$

In conclusion, it is apparently possible to achieve major reductions in admissions and deaths of patients with heart failure by implementing post-discharge interventions delivering patient education and symptom self management. These interventions appear to be particularly effective when they are at least partly delivered in a patient's own home through visits, telephone calls, or more advanced televideo techniques. 


\section{ACKNOWLEDGEMENTS}

We thank Jessie McGowan for her kind help searching the Cochrane Effective Practice and Organisation of Care Study Registry. We also thank the following authors for providing further data from their trials or publications: Brian Bixby (for Mary Naylor), Soccorso Capomolla, John Cleland, Alison Coletta, Inger Ekman, Wendy Gattis, Larry Goodyer, Margaret Harrison, Tiny Jaarsma, Anthony Jerant, Harlan Krumholz, Michael Rich, Barbara Riegel, and Anna Strömberg. RH was funded by MRC as a research fellow during the course of this review.

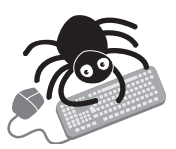

To view tables 1 and 2 visit the Heart website-http:// www.heartjinl.com/supplemental.

\section{Authors' affiliations}

R Holland, J Battersby, I Harvey, E Lenaghan, J Smith, L Hay, School of Medicine, Health Policy and Practice, University of East Anglia, Norwich, Norfolk, UK

\section{REFERENCES}

1 Davies M, Hobbs F, Davis R, et al. Prevalence of left-ventricular systolic dysfunction and heart failure in the echocardiographic heart of England screening study: a population based study. Lancet 2001;358:439-44.

2 Stewart S, Jenkins A, Buchan S, et al. The current cost of heart failure to the National Health Service in the UK. Eur J Heart Fail 2002;4:361-71.

3 Jessup M, Brozena S. Heart failure. N Engl J Med 2003;348:2007-18.

4 Horowitz JD. Home-based intervention: the next step in treatment of chronic heart failure? Eur Heart J 2000;21:1807-9.

5 McAlister FA, Lawson FM, Teo KK, et al. A systematic review of randomized trials of disease management programs in heart failure. Am J Med $2001 ; 110: 378-84$.

6 Phillips CO, Wright SM, Kern DE, et al. Comprehensive discharge planning with postdischarge support for older patients with congestive heart failure: a meta-analysis. JAMA 2004;291:1358-67.

7 Rich MW. Heart failure disease management: a critical review. J Card Fail 1999:5:64-75

8 Riegel B, Carlson B, Kopp Z, et al. Effect of a standardized nurse casemanagement telephone intervention on resource use in patients with chronic heart failure. Arch Intern Med 2002;162:705-12.

9 Juni P, Altman DG, Egger M. Assessing the quality of randomised controlled trials. In: Systematic reviews in health care: meta-analysis in context. London: BMJ Publishing Group, 2001.

10 Centre for Reviews \& Dissemination. Undertaking systematic reviews of research on effectiveness. York: University of York, 2001.

11 Holland R, Lenaghan E, Harvey I, et al. Does home based medication review keep older people out of hospital? The HOMER randomised controlled trial. $B M J$ (in press).

12 Goodyer LI, Miskelly F, Milligan P. Does encouraging good compliance improve patients' clinical condition in heart failure? Br J Clin Pract 1995;49:173-6

13 Coletta AP, Louis AA, Clark AL, et al. Clinical trials update from the European Society of Cardiology: CARMEN, EARTH, OPTIMAAL, ACE, TEN-HMS MAGIC, SOLVD-X and PATH-CHF II. Eur J Heart Fail 2002;4:661-6.

14 Rainville EC. Impact of pharmacist interventions on hospital readmissions for heart failure. Am J Health Syst Pharm 1999;56:1339-42.

15 Jaarsma T, Halfens R, Huijer Abu-Saad $H$, et al. Effects of education and support on self-care and resource utilization in patients with heart failure. Eur Heart J 1999;20:673-82.

16 Rich MW, Beckham V, Wittenberg C, et al. A multidisciplinary intervention to prevent the readmission of elderly patients with congestive heart failure. N Engl J Med 1995:333:1190-5.

17 Ekman I, Andersson B, Ehnfors M, et al. Feasibility of a nurse-monitored, outpatient-care programme for elderly patients with moderate-to-severe, chronic heart failure. Eur Heart J 1998; 19:1254-60.

18 Krumholz HM, Amatruda J, Smith GL, et al. Randomized trial of an education and support intervention to prevent readmission of patients with heart failure. J Am Coll Cardiol 2002;39:83-9.

19 Rich MW, Vinson JM, Sperry JC, et al. Prevention of readmission in elderly patients with congestive heart failure: results of a prospective, randomized pilot study. J Gen Intern Med 1993;8:585-90.
20 Gattis WA, Hasselblad V, Whellan DJ, et al. Reduction in heart failure events by the addition of a clinical pharmacist to the heart failure management team: results of the pharmacist in heart failure assessment recommendation and monitoring (PHARM) study. Arch Intern Med 1999;159:1939-45.

21 Grancelli $\mathrm{H}$, Varini S, Ferrante D, et al. Randomized trial of telephone intervention in chronic heart failure (DIAL): study design and preliminary observations. J Card Fail 2003;9:172-9.

22 De Lusignan $S$, Wells $S$, Johnson $\mathrm{P}$, et al. Compliance and effectiveness of 1 year's home telemonitoring: the report of a pilot study of patients with chronic heart failure. Eur J Heart Fail 2001;3:723-30.

23 Blue L, Lang E, McMurray JJ, et al. Randomised controlled trial of specialist nurse intervention in heart failure. BMJ 2001;323:715-8.

24 Doughty RN, Wright SP, Pearl A, et al. Randomized, controlled trial of integrated heart failure management: the Auckland heart failure management study. Eur Heart J 2002;23:139-46.

25 Kasper EK, Gerstenblith G, Hefter G, et al. A randomized trial of the efficacy of multidisciplinary care in heart failure outpatients at high risk of hospital readmission. J Am Coll Cardiol 2002;39:471-80.

26 Naylor MD, Brooten DA, Campbell RL, et al. Transitional care of older adults hospitalized with heart failure: a randomized, controlled trial. J Am Geriatr Soc 2004;52:675-84.

27 Weinberger M, Oddone EZ, Henderson WG. Does increased access to primary care reduce hospital readmissions? Veterans Affairs Cooperative Study Group on Primary Care and Hospital Readmission. N Engl J Med 1996; 334:1441-7.

28 Serxner S, Miyaji M, Jeffords J. Congestive heart failure disease management study: a patient education intervention. Prev Manage Congest Heart Fail 1998:4:23-8

29 Philbin EF, Rocco TA, Lindenmuth NW, et al. The results of a randomized trial of a quality improvement intervention in the care of patients with heart failure. The MISCHF Study Investigators. Am J Med 2000;109:443-9.

30 Varma S, McElnay JC, Hughes CM, et al. Pharmaceutical care of patients with congestive heart failure: interventions and outcomes. Pharmacotherapy 1999:19:860-9.

31 Capomolla S, Febo O, Ceresa M, et al. Cost/utility ratio in chronic heart failure: comparison between heart failure management program delivered by day-hospital and usual care. J Am Coll Cardiol 2002;40:1259-66.

32 Stewart S, Pearson S, Horowitz JD. Effects of a home-based intervention among patients with congestive heart failure discharged from acute hospital care. Arch Intern Med 1998;158:1067-72.

33 Bouvy ML, Heerdink ER, Urquhart J, et al. Effect of a pharmacist-led intervention on diuretic compliance in heart failure patients: a randomized controlled study. J Card Fail 2003;9:404-11.

34 Cline CM, Israelsson BY, Willenheimer RB, et al. Cost effective management programme for heart failure reduces hospitalisation. Heart 1998;80:442-6.

35 Goldberg LR, Piette JD, Walsh MN, et al. Randomized trial of a daily electronic home monAS, Levinsky SK, Sargent J, et al. Case management in a heterogeneous congestive itoring system in patients with advanced heart failure: the weight monitoring in heart failure (WHARF) trial, Am Heart J 2003; 146:705-12.

36 Laramee AS, Levinsky SK, Sargent J, et al. Case management in a heterogeneous congestive heart failure population: a randomized controlled trial. Arch Intern Med 2003;163:809-17.

37 McDonald K, Ledwidge M, Cahill J, et al. Elimination of early rehospitalization in a randomized, controlled trial of multidisciplinary care in a high-risk, elderly heart failure population: the potential contributions of specialist care, clinical stability and optimal angiotensin-converting enzyme inhibitor dose at discharge. Eur J Heart Fail 2001;3:209-15.

38 Stewart S, Marley JE, Horowitz JD. Effects of a multidisciplinary, home-based intervention on unplanned readmissions and survival among patients with chronic congestive heart failure: a randomised controlled study. Lancet 1999:354:1077-83.

39 Stromberg A, Martensson J, Fridlund B, et al. Nurse-led heart failure clinics improve survival and self-care behaviour in patients with heart failure: results from a prospective, randomised trial. Eur Heart J 2003;24:1014-23.

40 Garg R, Yusuf S. Overview of randomized trials of angiotensin-converting enzyme inhibitors on mortality and morbidity in patients with heart failure. Collaborative Group on ACE Inhibitor Trials. JAMA 1995;273:1450-6.

41 Benatar D, Bondmass M, Ghitelman J, et al. Outcomes of chronic heart failure. Arch Intern Med 2003;163:347-52.

42 Jerant AF, Azari R, Nesbitt TS. Reducing the cost of frequent hospita admissions for congestive heart failure: a randomized trial of a home telecare intervention. Med Care 2001;39:1234-45.

43 Harrison MB, Browne GB, Roberts J, et al. Quality of life of individuals with heart failure: a randomized trial of the effectiveness of two models of hospitalto-home transition. Med Care 2002;40:271-82.

44 Jaarsma T, Van Der Wal MH, Hogenhuis J, et al. Design and methodology of the $\mathrm{COACH}$ study: a multicenter randomised coordinating study evaluating outcomes of advising and counselling in heart failure. Eur $J$ Heart Fail 2004;6:227-33. 